Clinical Report

\title{
Clinical Training in Department of General Dentistry at Tokyo Dental College Chiba Hospital
}

Toshiko Sugiyama, Masatake Tsunoda, Toshiyuki Takahashi, Daiki Yamakura, Yu-kou Miyashita, Ryou Adachi, Kazuki Morinaga*, Akiyo Itou**, Chikahiro Haruyama***, Tomotake Takeda**** and Kazunori Nakajima****

Chiba Hospital General Dentistry, Tokyo Dental College, 1-2-2 Masago, Mihama-ku, Chiba 261-8502, Japan

* Department of Endodontics, Pulp and Periapical Biology, Tokyo Dental College, 1-2-2 Masago, Mihama-ku, Chiba 261-8502, Japan

** Department of Periodontology, Tokyo Dental College, 1-2-2 Masago, Mihama-ku, Chiba 261-8502, Japan

*** Department of Operative Dentistry, Tokyo Dental College, 1-2-2 Masago, Mihama-ku, Chiba 261-8502, Japan

**** Department of Sports Dentistry, Tokyo Dental College, 1-2-2 Masago, Mihama-ku, Chiba 261-8502, Japan

Received 12 December, 2007/Accepted for publication 15 January, 2008

\begin{abstract}
A compulsory postgraduate clinical training program was established in April 2006 in Japan, and an applicants-only postgraduate training program 9 years ago at Tokyo Dental College. In addition, a training program was also established in the Department of General Dentistry at Tokyo Dental College Chiba Hospital in April 2002. The curriculum consists of training in the outpatient clinic and the following: 1) clinical training (preparation of written treatment plans, simulation practice, submission of evaluation sheets, and submission of training journals), 2) tutorials, and 3) case reports. In 1), trainees write treatment plans for new patients, discuss them with their instructor, perform simulation practice using dummies based on those discussions, submit evaluation sheets and training journals concerning treatment, and receive their instructor's assessment. In 2), trainees are divided into small groups, independently study themes they have chosen, and present the results. In 3), they orally report cases they have treated and receive evaluation by other trainees and instructors in general discussion meetings. In addition, a course was also established at the Department of General Dentistry, Tokyo Dental College Chiba Hospital in April 2002. We report the training curriculum of this course.
\end{abstract}

Key words: Clinical trainee dentist-Postgraduate dentist-Clinical training system Department of General Dentistry—Tokyo Dental College Chiba Hospital

An abstract of this paper was presented at the 282nd General Meeting of Tokyo Dental College Society (November 4, 2006; Chiba). 


\section{Introduction}

A compulsory postgraduate clinical training was established in April 2006 in Japan. The purpose of the program was to cultivate well-rounded dentists with an understanding of patient-centered holistic medicine, and a comprehensive mastery of the basic techniques of dentistry required by all dentists as the first step of lifelong training ${ }^{1)}$. In Tokyo Dental College, a postgraduate training system for applicants only was established 9 years ago. The results of this training program, including tutorials and case reports are described in Report on the Postgraduate Dentists Clinical Training in General Dentistry ${ }^{1-3)}$. In addition, a course was also established at the Department of General Dentistry, Tokyo Dental College Chiba Hospital in April 2002. We report the training curriculum of this course.

\section{Methods}

Training is divided into 3 units, each with a different program of study. The 3 units cover training in general dentistry, specialized training, and training in cooperation-type clinical training institutions, respectively. The course consists of treatment at the outpatient clinic and the following: 1) clinical training (preparation of written treatment plans, simulation practices, submission of evaluation sheets, and submission of training journals), 2) tutorials, and 3) case reports.

\section{Clinical training}

1) Preparation of written treatment plans

With new patients, problems are clarified and classified as active or non-active based on X-ray films, intraoral photos, dental casts for examination, and findings of various examinations. Treatment principles and procedures are planned, and treatment plans are written. Trainees fully discuss the written treatment plans with their instructor and perform treatment (Fig. 1).

2) Simulation practices

At present, there are 10 dummies for simu-

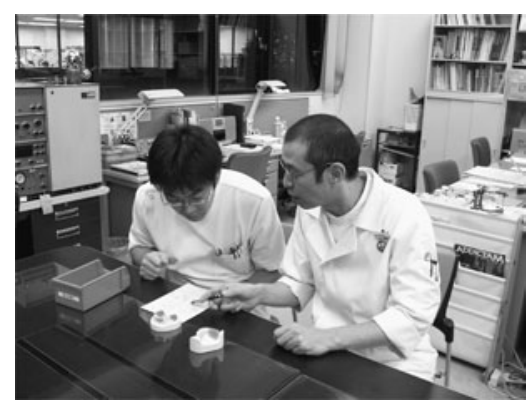

Fig. 1 Discussion scene

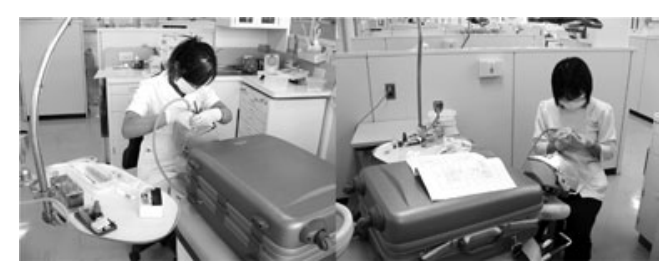

Fig. 2 Simulation training

lation practice in the Department of General Dentistry. Before carrying out a written treatment plan, that treatment is simulated using a dummy, and the work is checked by an instructor. In their free time, trainees are required to practice using jaw models according to guidelines on cavity/abutment tooth preparation contained in a self-learning manual, with each step being assessed by their instructor. In addition, they practice access cavity preparation, root canal enlargement, and root canal filling using plaster models with inserted natural teeth (Fig. 2).

3) Submission of evaluation sheets

The evaluation sheet contains a number of items, including those related to basic skills for medical interviews and treatment, oral health guidance, root canal treatment, periodontal treatment, preservation/restoration, prosthodontic treatment, crown/bridge prosthesis, tooth extraction, and dental laboratory procedures. Trainees fill in the sheet, check whether each case has been appropriately treated according to the contents of the sheet, rate themselves, describe their feelings and problems, and receive the results of their instructor's overall evaluation and comments. 


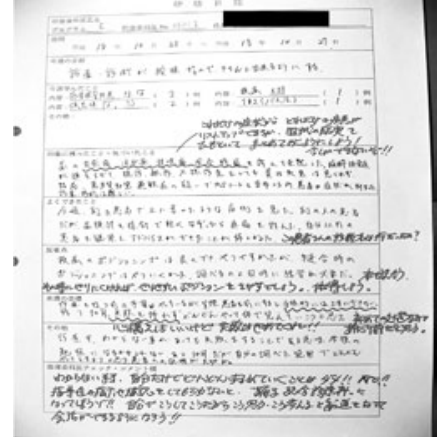

Fig. 3 Training journal

4) Submission of training journals

At the end of each week, trainees describe the goal of the week, what they have learned, what they were impressed by, noticed, could successfully perform, or regret, the goal of the following week, and any other items in the journal. This is then submitted to their instructor, who writes comments and returns it to the trainees (Fig. 3).

\section{Tutorials}

Trainees are divided into small groups (46 trainees in each group); each group chooses an interesting theme, studies, and then presents the results (Fig. 4). Each group studies themes of interest chosen by themselves and orally presents the results. This presentation is evaluated using a 5-grade system (very good, good, average, poor, very poor) in terms of the following items; 1) data were presented in a form easy to understand; 2) theme was clear and interesting; 3) learned items were well-summarized; 4) contents of report were well conveyed by oral presentation; and 5) discussion was smoothly advanced. Questions and opinions are also expressed, and the results of the evaluation are returned to each group. In this way, trainees can be alerted to problems they have overlooked, so that they will be aware of them in the future.

\section{Case reports}

Each trainee report on the examination, diagnosis, and treatment of patients, and

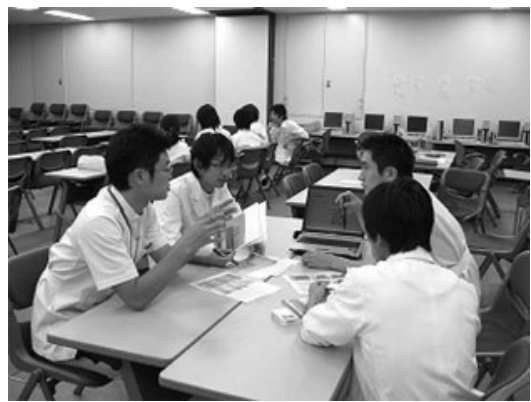

Fig. 4 Group discussion

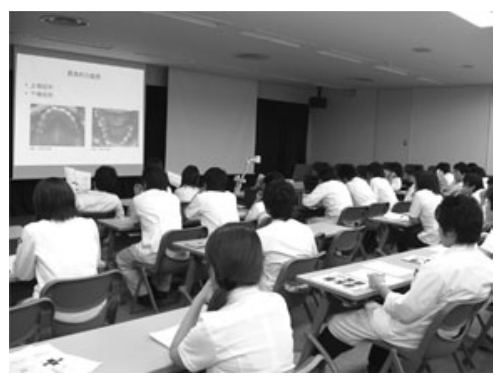

Fig. 5 Case report

other trainees evaluate that report according to the following items using a 5-grade system similar to that used in tutorials; 1 ) intraoral films, dental casts, and examination materials accurately obtained; 2) findings obtained from intraoral photos, models, and examination materials; 3) problem accurately described; 4) appropriate treatment plan devised;5) treatment course visually explained; and 6) data presented in a form easy to understand. In addition, opinions and confirmation are expressed, and the results of the evaluation are returned to each trainee. Thus, comments that cannot be given during the short presentation time can also be given, allowing the trainee to become aware of any problems and take them into consideration in future planning of treatment. In the case reports, the trainees report on the findings of examination at initial visit in each patient, diagnosis, and treatment course. This is then discussed among all the trainees (Fig. 5). 


\section{Results and Discussion}

\section{Clinical training}

Preparation of written treatment plans provides training in interpreting X-ray films, collecting accurate information from intraoral photos and dental casts for examination, interpreting findings of various examinations, and clarifying problems. Discussion with the instructor on the treatment planned by the trainees allows them to correct any misunderstanding, supplement inadequate items, and identify the correct approach to be taken.

Before carrying out actual treatment, simulation using a dummy relieves anxiety about skills, promoting confidence. Questions can also be answered before treatment.

Trainees can clarify what they can and cannot do on the evaluation sheets, and confirm the reasons for this and points requiring improvement based on their instructor's comments and discussion with the instructor. Trainees can also re-confirm these matters by re-evaluating the evaluation sheet later on.

By submitting a weekly training journals, the trainees provide information on the number of patients they have treated and the conditions encountered. When a trainee has misinterpreted some learning material, been impressed by something, or wishes to clarify something in the journal, their instructor can correct that misinterpretation, give positive feedback on successful points, and make comments, including point-by-point advice. Thus, this journal is useful in improving skills and providing feedback.

\section{Tutorials}

The themes chosen were endodontics $(21 \%)$, periodontology $(21 \%)$, operative dentistry (19\%), fixed prosthodontics (17\%), removable prosthodontics $(9 \%)$, and others $(13 \%)$. Contents included measures for prevention of perforation, selection of root canal treatment agents, halitosis, the basis of SRP (scaling root playing), prevention of secondary caries in metal inlay restoration, and an esthetic prosthesis. Each group not only orally presents reports, but also listens to reports by other groups. These reports are then discussed by the trainees and instructors together, which promotes sharing of themes and knowledge.

\section{Case reports}

The themes of reports were periodontology (26\%), fixed prosthodontics (26\%), operative dentistry $(17 \%)$, removable prosthodontics $(13 \%)$, endodontics (11\%), and others (6\%).

All submitted documents such as selfevaluation sheets and training journals are returned, together with instructors' comments to trainees, and collected as portfolios for feedback. We evaluated the curriculum provided at the Department of General Dentistry, Tokyo Dental College Chiba Hospital since 2002. Further studies are necessary to improve training and establish appropriate evaluation methods.

\section{Acknowledgements}

We would like to thank Associate Professor Jeremy Williams, Tokyo Dental College, for his assistance with the English of this manuscript.

\section{References}

1) Tokyo Dental College Chiba Hospital (2003) Report on the Postgraduate Dentists Clinical Training in General Dentistry in 2003rd. (in Japanese)

2) Tokyo Dental College Chiba Hospital (2004) Report on the Postgraduate Dentists Clinical Training in General Dentistry in 2004th. (in Japanese)

3) Tokyo Dental College Chiba Hospital (2005) Report on the Postgraduate Dentists Clinical Training in General Dentistry in 2005th. (in Japanese)

Reprint requests to:

Dr. Toshiko Sugiyama

General Dentistry,

Tokyo Dental College, 1-2-2, Masago, Mihama-ku, Chiba 260-8502, Japan, E-mail: tosugiya@tdc.ac.jp 